\title{
Additive Manufactured Metal-Only Modulated Metasurface Antennas
}

\author{
David González-Ovejero, Senior Member, IEEE, Nacer Chahat, Senior Member, IEEE, \\ Ronan Sauleau, Fellow, IEEE, Goutam Chattopadhyay, Fellow, IEEE, Stefano Maci, Fellow, IEEE, \\ and Mauro Ettorre, Senior Member, IEEE
}

\begin{abstract}
In metasurface (MTS) antennas, a surface-wave (SW) is gradually transformed into a leaky-wave (LW), owing to the SW interaction with a reactance tensor periodically modulated in the aperture plane. In such antennas, the modulated reactance is typically obtained by sub-wavelength patches printed on a dielectric substrate. As an alternative technology, we propose the use of a class of metal-only MTSs in the synthesis of the reactance tensor. The absence of dielectric is useful to withstand harsh environments in space applications. The proposed element consists of a metallic cylinder with elliptical cross-section, placed on a ground plane and arranged in a square lattice with subwavelength unit-cells. This structure is well suited for fabrication by metal additive manufacturing. We have applied the metal cylinders in the design of a right-handed circularly polarized (RHCP) antenna, with broadside pencil beam at Ka band. The obtained performance has been verified by full-wave simulations. A prototype has been successfully manufactured and measured, showing a good performance.
\end{abstract}

Index Terms - circular polarization, Ka band, leaky waves, metasurfaces, millimeter waves, surface waves.

\section{INTRODUCTION}

$\mathbf{I}$ $\mathrm{N}$ this paper, we present a new type of low-profile high-gain metasurface (MTS) antenna. The proposed structure (see Fig. 1) is composed of metal-only sub-wavelength elements, well suited to fabrication by additive manufacturing. The MTS element consists in a metallic cylinder with elliptical crosssection, grown on a ground plane and arranged in a subwavelength square lattice (see the bottom-right inset in Fig. 1). The elliptical cylinders exhibit different orientations, heights and ratios between the major and minor axes of the ellipse across the aperture, which allows one to control the anisotropy and modulation of the equivalent reactance. The interface between the end point of the metallic cylinders and free-space

Manuscript received May 16, 2018; revised August 21, 2018; accepted August 21, 2018. This publication has been supported by the European Union through the European Regional Development Fund (ERDF), and by the french region of Brittany, Ministry of Higher Education and Research, Rennes Métropole and Conseil Départemental 35, through the CPER Project STIC \& Ondes. A part of the work has been carried out at the Jet Propulsion Laboratory, California Institute of Technology, Pasadena, CA, USA, under a contract with National Aeronautics and Space Administration. It was sponsored through an internal Research and Technology Development funds.

D. González-Ovejero, R. Sauleau and M. Ettorre are with the Institut d'Electronique et de Télécommunications de Rennes - IETR, UMR CNRS 6164, Université de Rennes 1, 35042 Rennes, France (e-mail: david.gonzalezovejero@univ-rennes1.fr).

N. Chahat and G. Chattopadhyay are with the Jet Propulsion Laboratory, California Institute of Technology, Pasadena, CA 91109, USA (e-mail: goutam.chattopadhyay@jpl.nasa.gov).

S. Maci is with Department of Information Engineering and Mathematics, University of Siena, Italy (e-mail: macis@dii.unisi.it).

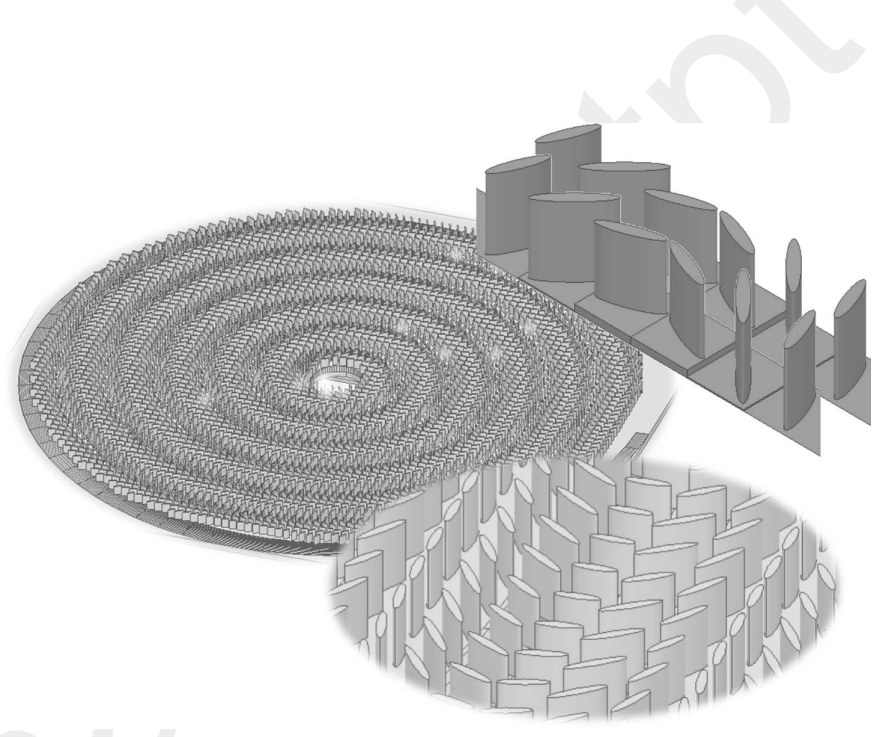

Fig. 1. CAD model of a metal-only MTS antenna consisting of subwavelength elliptical cylinders. The right-bottom inset shows a detail of the textured surface, whereas the top one shows the height variation of the cylinders obtained by stepping the ground.

is planar, whereas the base of the cylinders vary in height in order to modulate the reactance profile (see the top inset in Fig. 1).

The development of medium and high-gain telecommunications antennas is of crucial importance in any spacecraft. The most common solutions to obtain high to very-high gains in satellite systems are reflector antennas. However, reflectors might not be the best option when a low-profile is required. Phased-arrays constitute a versatile alternative, but they require complex feeding networks and their Rx/Tx modules are costly and energy-hungry. Even if a reduction of weight, volume, complexity and cost is always desirable, parabolic reflectors and phased-array antennas are particularly difficult to adapt to SmallSats and CubeSats, whose number of launches keeps increasing [1].

Significant efforts have been carried out recently to develop compact high-gain antennas for space. Among the most noteworthy solutions, one can find deployable reflectarrays [2] and meshed deployable reflectors [3]. Despite the elegance of deployable antennas, the risk associated to the deployment of both the feed and the mesh reflector or the reflectarray panels calls for alternative solutions. One possibility to get rid of the reflector deployment consists in integrating planar antennas on the spacecraft chassis. Another desirable characteristic lies in having the source on the aperture plane, so one can also eliminate the need of deploying the feed. Along these lines, 
it is possible to use corrugations around a rectangular subwavelength slot in order to get either a broadside pencil beam with concentric rings [4] or a tilted one with centershifted grooves [5]. The latter structures are low profile and can be readily integrated in the satellite chassis. However, the amplitude taper of the field is not addressed, and its polarization is forced by the source. Conversely, it has been shown that planar MTS antennas [6]-[12] provide an accurate control of the aperture fields, while preserving a low profile, low weight and a feed embedded in the aperture plane. This feed launches a dominantly transverse-magnetic (TM) surfacewave $(\mathrm{SW})$. The SW interaction with a periodically modulated surface reactance results in the (-1) indexed Floquet mode entering the visible region. The power is gradually radiated owing to this SW to leaky-wave (LW) transformation. As in the corrugated structures, the feed is embedded in the MTS, so no protruding feed or sub-reflector is required; this is an advantage with respect to other types of printed antennas like reflectarrays. Moreover, by using MTSs one can taper the aperture field and easily handle the polarization of the radiated fields.

Recent developments on the modeling of MTS antennas allow one to carry out an accurate control of the amplitude and phase of the aperture field with sub-wavelength patches printed on a grounded dielectric substrate [10]. However, the use of dielectric substrates might be limiting to withstand harsh environments in space applications [13], [14]. This includes large thermal ranges and high radiation levels. A metal-only design (e.g. aluminum) will be less susceptible to thermal variation and will not suffer for dielectric property change due to high level of radiation. The elimination of dielectric eliminates the possibility of electrostatic discharge, the hysteresis losses and the uncertainty in the dielectric constant (thus improving tuning accuracy).

A scalar reactance synthesized by an array of metallic cylinders placed on a ground plane has been used in [15], [16]. In the latter structure, which resembles a Fakir's bed of nails, it is possible to tune the inductance by changing the height of the cylinders. However, this geometry fails in providing a space-dependent anisotropic reactance. It is thus necessary to introduce additional degrees of freedom in order to model the anisotropic boundary condition, a key step to control the polarization. To that end, we propose fully metallic cylinders with elliptical cross-section (see the insets in Fig. 1). The elliptical cylinders allow one to exert the same control of the aperture field as the one already demonstrated with printed patches and can be easily fabricated by additive manufacturing. This technology has been recently used in the prototyping of dielectric MTS antennas [17] and lenses [18].

The paper is organized as follows. The synthesis of modulated MTS antennas is recalled in Section II. Section III describes the metallic MTS element and the way of retrieving the equivalent reactance from the full-wave analysis of the periodic structure that locally fits the antenna geometry. Section IV presents a design example, including the MTS layer and the SW launcher. Section V describes the fabrication process and presents the experimental results. Finally, Section VI draws conclusions.

\section{Modulated MTS ANTEnNAS}

In the synthesis problem, the goal is to find a structure able to generate an objective aperture field $\mathbf{E}_{A}$. We consider circular antennas of radius $R$, which present a planar interface with free-space at the $z=0$ plane of a Cartesian reference system with coordinates $(x, y, z)$ and unit vectors $(\hat{\mathbf{x}}, \hat{\mathbf{y}}, \hat{\mathbf{z}})$. Throughout this paper, vectors will be denoted by bold characters, unit vectors by bold characters with a caret, and tensors by bold characters underlined by double bars. An $\exp (\mathrm{j} \omega t)$ time dependence, where $\omega$ is the angular frequency, is assumed and suppressed. For convenience, we define the observation point on the aperture as $\boldsymbol{\rho}=\rho \cos \phi \hat{\mathbf{x}}+\rho \sin \phi \hat{\mathbf{y}}$ in a cylindrical coordinate $(\rho, \phi)$ system with unit vectors $(\hat{\boldsymbol{\rho}}, \hat{\boldsymbol{\phi}})$.

In the first instance, let us represent the periodically modulated MTS by the $\underline{\mathbf{X}}(\boldsymbol{\rho})$ tensor, which relates the total tangential electric and tangential magnetic fields on the MTS aperture as

$$
\left.\mathbf{E}_{t}\right|_{z=0^{+}}=\mathrm{j} \underline{\underline{\mathbf{X}}}(\boldsymbol{\rho}) \cdot \hat{\mathbf{z}} \times\left.\mathbf{H}_{t}\right|_{z=0^{+}}
$$

where we assume $\underline{\underline{\mathbf{X}}}(\boldsymbol{\rho})=\underline{\underline{\mathbf{X}}}(\boldsymbol{\rho}+p \hat{\rho})$, with $p$ being the period of the modulation. The fields in (1) are evaluated at the upper interface and $\hat{\mathbf{z}}$ is the normal to the MTS plane. Equation (1) describes an impenetrable boundary condition (BC) [19] $[20,(4)][21,(2.28)]$, as opposed to the penetrable [20] or sheet transition BCs [22], [23], which relate the fields on both sides of the MTS. The former will be used throughout this paper, whereas the latter is preferred in the synthesis of MTS antennas consisting of sub-wavelength printed patches [10]. Assuming that a source at the origin launches a cylindrical $\mathrm{SW}$ with wave-number $\beta_{s w}$, the total aperture field $\mathbf{E}_{t}$ on the impenetrable $\mathrm{BC}$ reads as

$$
\mathbf{E}_{t}=I_{T M} \mathrm{j} \underline{\underline{\mathbf{X}}}(\boldsymbol{\rho}) \cdot \hat{\boldsymbol{\rho}} \mathrm{H}_{1}^{(2)}\left(\left(\beta_{s w}-\mathrm{j} \alpha\right) \rho\right)
$$

where $I_{T M}$ is the complex excitation coefficient of the SW, and $\mathrm{H}_{1}^{(2)}(\cdot)$ is the Hankel function of the second kind and first order. The presence in (2) of the imaginary part $\alpha$ takes into account the radiation by leakage. The SW wave-number $\beta_{s w}$ is related with the average value of the $\rho$ component of $\underline{\underline{\mathbf{X}}}$ by $\beta_{s w}=k \sqrt{1+\left(X_{0} / \zeta\right)^{2}}$, where $k$ and $\zeta$ are the free-space wave-number and impedance, respectively.

The aperture fields $\mathbf{E}_{A}$ required to obtain a right-handed circularly polarized (RHCP) and a linearly polarized (LP) broadside pencil beam are

$$
\begin{aligned}
& \mathbf{E}_{A}=\mathrm{E}_{0}[\hat{\mathbf{x}}+\mathrm{j} \hat{\mathbf{y}}] \sqrt{\frac{2}{\pi \beta_{s w} \rho}} \mathrm{e}^{-\alpha \rho} \mathrm{U}_{A} \\
& \mathbf{E}_{A}=\mathrm{E}_{0} \hat{\mathbf{x}} \sqrt{\frac{2}{\pi \beta_{s w} \rho}} \mathrm{e}^{-\alpha \rho} \mathrm{U}_{A}
\end{aligned}
$$

respectively, where $\mathrm{E}_{0}$ is the field magnitude and $\mathrm{U}_{A}$ is a step function that equals one inside the aperture and is 0 elsewhere.

In order to find $\underline{\underline{X}}$, we identify the objective aperture field $\mathbf{E}_{A}$ with the -1 mode contribution of $\mathbf{E}_{t}$ in (2) [8], thus obtaining

$$
\underline{\underline{\mathbf{X}}}(\boldsymbol{\rho}) \cdot\left\{\begin{array}{c}
\hat{\boldsymbol{\rho}} \\
\hat{\boldsymbol{\phi}}
\end{array}\right\}=\left[\mathrm{X}_{0}\left\{\begin{array}{c}
\hat{\boldsymbol{\rho}} \\
\hat{\boldsymbol{\phi}}
\end{array}\right\} \pm 2 \operatorname{Im}\left(\frac{\mathbf{E}_{A}}{I_{T M} \mathrm{H}_{1}^{(2)}\left(\beta_{s w} \rho\right)}\right)\right] \mathrm{U}_{A}
$$




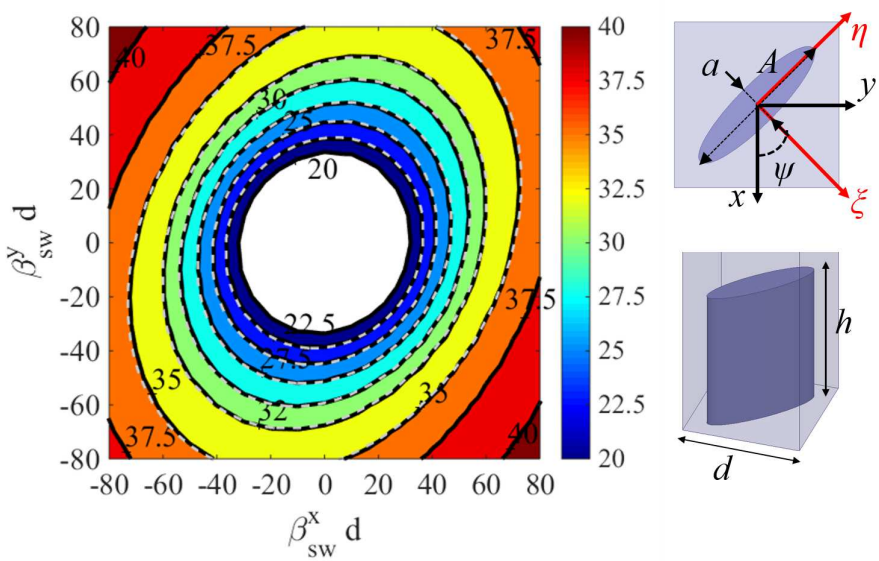

Fig. 2. Iso-frequency dispersion contours for the unit-cell depicted in the insets. The frequency values are in GHz. The unit-cell's side is $d=1.235 \mathrm{~mm}$, the height of the cylinder is $h=1.525 \mathrm{~mm}$, and the elliptical cross-section has minor axis $a$ equal to $240 \mu \mathrm{m}$ and major axis $A$ equal to $1.2 \mathrm{~mm}$.

where the period $p$ of the modulation is obtained from $\beta_{s w}=$ $2 \pi / p$ and the upper and lower signs correspond to the $\hat{\rho}$ and $\hat{\phi}$ components, respectively.

Using (3) in (5), along with the asymptotic expression of the Hankel function, and projecting $\underline{X} \cdot \hat{\rho}$ along $\hat{\rho}$ and $\hat{\phi}$ leads, for the RHCP aperture field, to

$$
\begin{aligned}
& \mathrm{X}_{\rho \rho}(\boldsymbol{\rho})=X_{0}[1+M \sin (2 \pi \rho / p-\phi)] \\
& \mathbf{X}_{\rho \phi}(\boldsymbol{\rho})=\mathbf{X}_{\phi \rho}(\boldsymbol{\rho})=X_{0} M \cos (2 \pi \rho / p-\phi) \\
& \mathbf{X}_{\phi \phi}(\boldsymbol{\rho})=X_{0}[1-M \sin (2 \pi \rho / p-\phi)]
\end{aligned}
$$

For the LP aperture field, one similarly gets

$$
\begin{aligned}
& \mathrm{X}_{\rho \rho}(\boldsymbol{\rho})=X_{0}[1+M \cos (\phi) \sin (2 \pi \rho / p)] \\
& \mathrm{X}_{\rho \phi}(\boldsymbol{\rho})=\mathrm{X}_{\phi \rho}(\boldsymbol{\rho})=-X_{0} M \sin (\phi) \sin (2 \pi \rho / p) \\
& \mathrm{X}_{\phi \phi}(\boldsymbol{\rho})=X_{0}[1-M \cos (\phi) \sin (2 \pi \rho / p)]
\end{aligned}
$$

where in both (6) and (7) the relation between $X_{0}$ and $p$ is $p=2 \pi /\left(k \sqrt{1+\left(X_{0} / \zeta\right)^{2}}\right)$. The above expressions include a modulation index $M$, which is a design parameter. In the following, we will assume $M$ constant, which implies that $\alpha$ is independent of $\boldsymbol{\rho}$ [8]. The use of a variable $M$ leads to a control of $\alpha$ along $\rho$, and can be used to taper the field amplitude along a desired profile [8], [10]. Thus, by using a variable $\alpha$ one could increase the aperture efficiency [24].

\section{Metallic MTS ELEMENTS}

Equation (6) and (7) provide the impenetrable reactance tensor which, upon interaction with a cylindrical SW, will generate the objective far-field. Once the expression of the space-dependent tensor $\underline{\mathbf{X}}$ has been computed on the aperture, one has to find a structure able to implement the entries of $\underline{\mathbf{X}}$. The unit-cell consists of metallic cylinders with elliptical cross-section (see the insets in Fig. 2). These cylinders have height $h$, orientation angle $\psi$, and present an elliptical crosssection with major axis $A$ and minor axis $a$.

The entries of $\underline{\mathbf{X}}$ for a given geometry are retrieved using an eigen-mode full-wave solver [25]. Based on a local periodicity assumption, the solution can be obtained for a unit-cell with periodic boundary conditions. For each pair of phase-shifts $\left(\psi_{x}, \psi_{y}\right)$ applied to the unit-cell in the $x$ and $y$ directions, one obtains the propagation frequency $f$ for the fundamental mode. The components of the transverse wave vector $\boldsymbol{\beta}_{\mathrm{sw}}=\beta_{\mathrm{sw}} \hat{\boldsymbol{\rho}}=\beta_{\mathrm{sw}}^{x} \hat{\mathbf{x}}+\beta_{\mathrm{sw}}^{y} \hat{\mathbf{y}}$ are obtained from the phases as $\beta_{\mathrm{sw}}^{x}=\psi_{x} / d$ and $\beta_{\mathrm{sw}}^{y}=\psi_{y} / d$. Fig. 2 shows the resulting dispersion map for an elliptical cylinder with $h=1.525 \mathrm{~mm}, a=240 \mu \mathrm{m}$ and $A=1.2 \mathrm{~mm}$, in a square unit cell with side $d=1.235 \mathrm{~mm}$. In turn, the solid black lines represent the iso-frequency dispersion curves defined as $f_{0}=f\left(\beta_{\mathrm{sw}}^{x}, \beta_{\mathrm{sw}}^{y}\right)$ [26], where $f_{0}$ is the design frequency. Then, the surface reactance tensor at $f_{0}$ can be retrieved from the corresponding iso-frequency dispersion curves by fitting, in the least square sense, the simulated data with [27, eq. 19]

$$
\begin{aligned}
& \left(-1+\mathbf{X}_{x x} \mathbf{X}_{y y}-\mathbf{X}_{x y} \mathbf{X}_{y x}\right) k \alpha_{z}+\left(\mathbf{X}_{x y}+\mathbf{X}_{y x}\right) \beta_{\mathrm{sw}}^{x} \beta_{\mathrm{sw}}^{y} \\
& +\left[\left(\mathbf{X}_{x x}+\mathbf{X}_{y y}\right) k^{2}-\mathbf{X}_{x x}\left(\beta_{\mathrm{sw}}^{y}\right)^{2}-\mathbf{X}_{y y}\left(\beta_{\mathrm{sw}}^{x}\right)^{2}\right]=0
\end{aligned}
$$

where $\alpha_{z}=\sqrt{\beta_{\mathrm{sw}}^{2}-k^{2}}$ and the reactance quantities $\mathrm{X}_{a b}$ in (8) are expressed in a Cartesian reference system. Equation (8) is derived by imposing the transverse resonance $\operatorname{det}\left(\underline{\underline{\mathbf{X}}}^{-1}+\underline{\underline{\mathbf{X}}}_{0}^{-1}\right)=0$, where $\underline{\underline{\mathbf{X}}}_{0}=\mathrm{j} \zeta\left(-\alpha_{z} / k \hat{\boldsymbol{\rho}} \hat{\boldsymbol{\rho}}+k / \alpha_{z} \hat{\boldsymbol{\phi}} \hat{\boldsymbol{\phi}}\right)$. By repeating the above procedure for each geometry, one can build a database which univocally links the geometry of the unit-cell with the entries of $\underline{\underline{\mathbf{X}}}$. Symmetries in the unit-cell geometry can be exploited to build the database. In this particular case, one can retrieve from $\underline{\underline{\mathbf{X}}}$ obtained for $\psi \in[0, \pi / 4]$ the values in the full $\psi \in[0,2 \pi[$ range.

Despite the possibility of reducing the number of calculations for some geometries, building a 2D map (like the one in Fig. 2) for each possible configuration leads to long computations. One can drastically reduce the computation time by aligning $\boldsymbol{\beta}_{s w}$ with the principal axes of the dispersion curve, which can be identified with the two orthogonal symmetry axes of the elliptical cylinder ( $\xi$ and $\eta$ in the top right inset of Fig. 2). After that, one can retrieve the principal values of the reactance tensor, $\mathrm{X}_{\xi}$ and $\mathrm{X}_{\eta}$, using the full-wave eigensolver. Then, the Cartesian components of $\underline{\mathbf{X}}$ can be written as $\underline{\underline{\mathbf{X}}}=\underline{\underline{\mathbf{R}}}(\psi)^{\mathrm{T}} \operatorname{diag}\left(\mathbf{X}_{\xi}, \mathbf{X}_{\eta}\right) \underline{\underline{\mathbf{R}}}(\psi)$, where $\psi$ is the orientation angle of the ellipse in Fig. 2 , T denotes the transpose, and $\underline{\underline{\mathbf{R}}}=\left[[\cos \psi, \sin \psi]^{\mathrm{T}} ;[-\sin \psi, \cos \psi]^{\mathrm{T}}\right]$. The corresponding iso-frequency dispersion curves (dashed lines in Fig. 2) are plotted using (8). This approximate procedure leads to accurate results, while reducing the database construction time by a factor of 8 . It has been shown in previous works that the same approximate procedure does not provide the same degree of accuracy for MTSs based on printed patches [26], [28].

Using the methodology in the paragraph above, one can build a database which relates the surface reactance tensor with the geometry of the unit-cell. Fig. 3 shows the values of $\mathrm{X}_{x x}$, $\mathrm{X}_{x y}$ and $\mathrm{X}_{y y}$ (Cartesian entries of $\underline{\underline{\mathbf{X}}}$ ) for different orientations, ratios $A / a$ and heights $h$ of the elliptical cylinders. It is important to note that, in order to guarantee the mechanical robustness of the final structure, we typically construct the database for $h / a$ ratios smaller than 10 .

\section{ANTENNA DESIGN}

Next, we present the design of two Ka-band antennas operating in the $31.8-32.3 \mathrm{GHz}$ deep space downlink band 


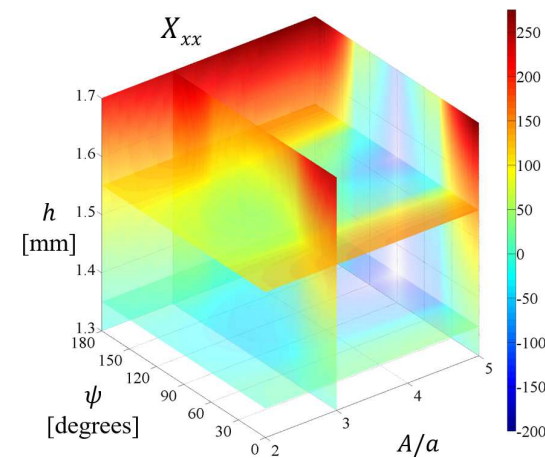

(a)

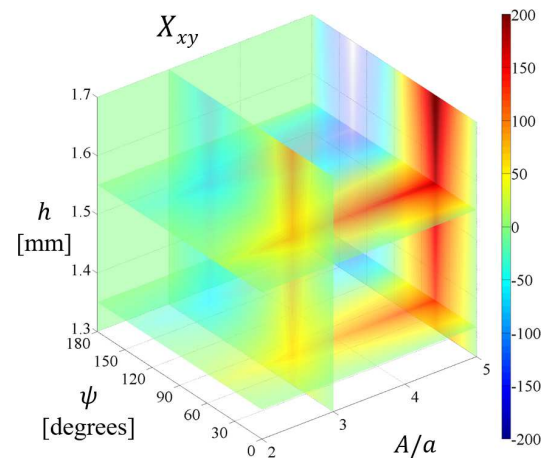

(b)

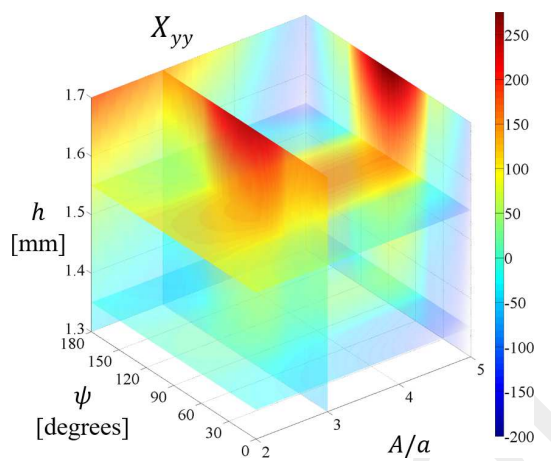

(c)

Fig. 3. Maps of (a) $\mathrm{X}_{x x}-X_{0}$, (b) $\mathrm{X}_{x y}$ and (c) $\mathrm{X}_{y y}-X_{0}$ as a function of the orientation angle $\psi$, the ratio $A / a$ and the height $h$ of the cylinder. The simulation frequency is $f_{0}=32 \mathrm{GHz}$, the unit-cell side is $d=1.235 \mathrm{~mm}$ and $X_{0}=0.8 \zeta$.

(space to Earth) [29]. The first design provides a circularly polarized broadside beam (6), while in the second one we target linear polarization (7). In the following subsections, the MTS synthesis and the feeding circuit are described.

\section{A. MTS synthesis}

In the implementation of (6) and (7) we have used $X_{0}=$ $0.8 \zeta, M=0.4, d=1.235 \mathrm{~mm}$ and $p=6 d$. The antenna radius is $R=5 \lambda_{0}$, where $\lambda_{0}$ is the free-space wavelength at $32 \mathrm{GHz}$. To synthesize the tensors in (6) and (7), the impedance surface is sampled on a regular Cartesian lattice, with the same unit-cell size $(d)$ as the database. Then, each impedance sample is implemented using a metallic pillar inside the corresponding lattice cell by retrieving its geometry from the database. Although (6) and (7) provide the entries of $\underline{\underline{X}}$ in cylindrical coordinates, they can be easily transformed to a Cartesian reference system by a rotation matrix. Then, one can establish a direct link with the values in the database (see Fig. 3). In both cases, the final structure consists of around 4500 elliptical cylinders with heights comprised between $1.325 \mathrm{~mm}$ and $1.525 \mathrm{~mm}$.

The far-field gain patterns have been computed at $32 \mathrm{GHz}$ using a full-wave solver [25]. Fig. 4a shows the RHCP broadside beam obtained with (6) in the $\phi=0^{\circ}, \phi=90^{\circ}$ and $\phi=45^{\circ}$ planes, whereas Fig. $4 \mathrm{~b}$ displays the LP broadside beam realized using (7). The peak gain for the RHCP antenna is $26.1 \mathrm{dBi}$, which implies a $40 \%$ aperture efficiency. In turn, the LP antenna presents a $25.6 \mathrm{dBi}$ peak gain and a $36.8 \%$ aperture efficiency. Larger aperture efficiencies can be obtained by tapering the modulation index and the corresponding $\alpha$. The simulated $-1 \mathrm{~dB}$ gain bandwidth is around $5 \%$ for both designs, we thus exceed the $1.6 \%$ bandwidth required for the Ka downlink band.

\section{B. Feeding circuit}

The antennas are fed by a circular waveguide excited in the $\mathrm{TM}_{01}$ first higher-order mode. The $\mathrm{TM}_{01}$ mode provides an efficient excitation on the MTS plane of a circularly symmetric TM SW mode, which matches (2) within a good approximation. This circular waveguide excitation has been preferred over a coaxial feeder (often used in printed elements
MTS design) owing to its better performance in terms of

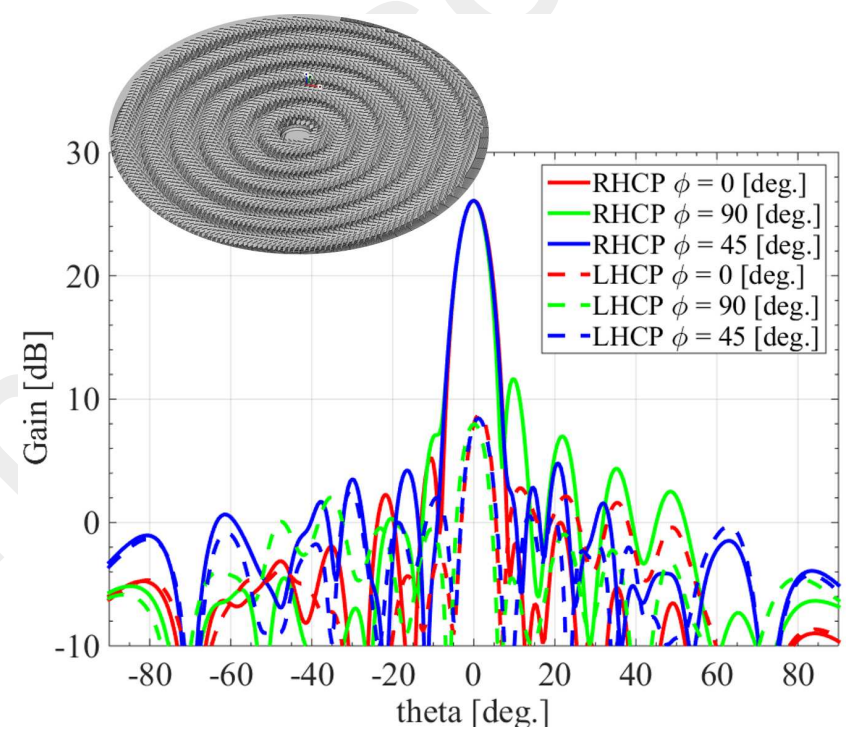

(a)

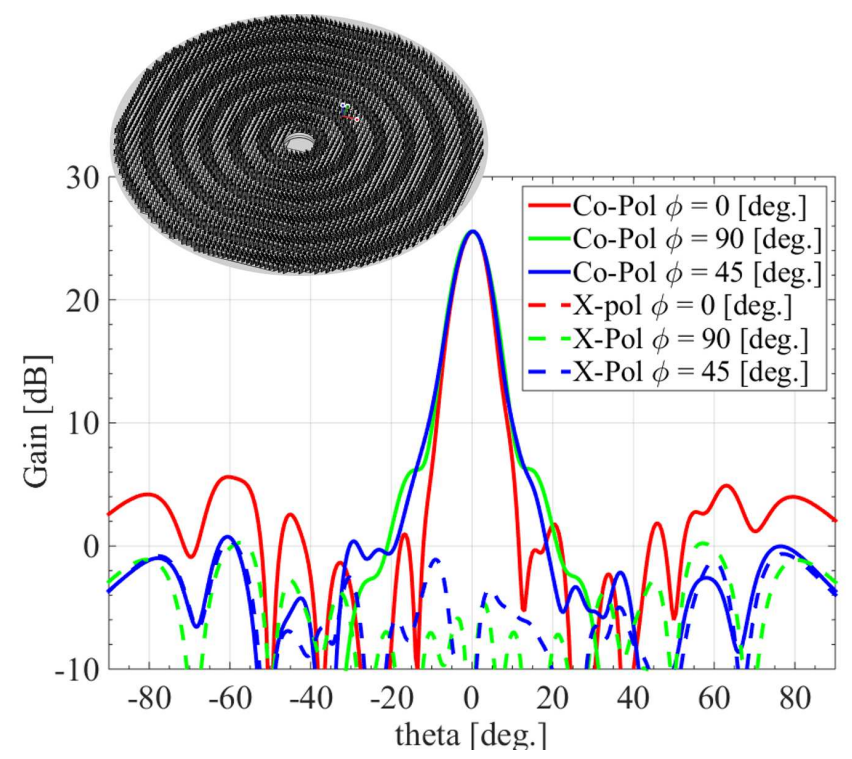

(b)

Fig. 4. Simulated radiation patterns for the (a) RHCP and the (b) linearly polarized MTS antenna with $R=5 \lambda_{0}$ at $32 \mathrm{GHz}$. 


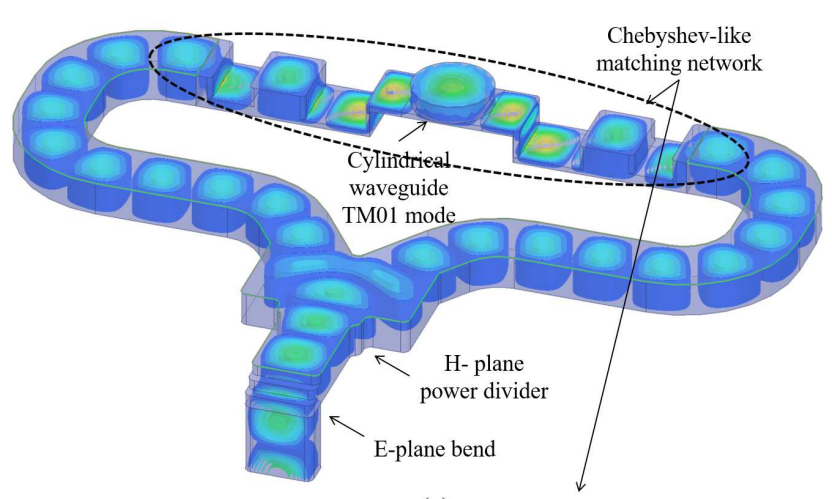

(a)

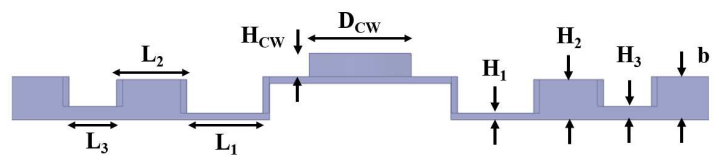

(b)

Fig. 5. (a) Feeding network constituted by an H-plane power divider in rectangular waveguide, each branch of the divider feeds symmetrically a circular waveguide in order to excite only its $\mathrm{TM}_{01}$ mode. (b) Geometry of the Chebyshev-like matching network longitudinal cross-section.

TABLE I

DIMENSIONS OF THE OPTIMIZED FEEDER

\begin{tabular}{lcccccc}
\hline Variable & $\mathrm{L}_{1}$ & $\mathrm{~L}_{2}$ & $\mathrm{~L}_{3}$ & $\mathrm{H}_{1}$ & $\mathrm{H}_{2}$ & $\mathrm{H}_{3}$ \\
\hline Value $[\mathrm{mm}]$ & 6.255 & 5.771 & 3.910 & 0.056 & 3.233 & 1.053 \\
\hline
\end{tabular}

losses and to the possibility of easily scaling in frequency the waveguides.

The complete structure, shown in Fig. 5, consists of an Hplane power divider in rectangular waveguide, which feeds two symmetric sections placed underneath the MTS. We choose to use the H-plane in order to obtain a low profile feeder. The two $\mathrm{TE}_{10}$ branches arrive to the circular waveguide on two sides. The symmetry of this geometry leads to the suppression of the fundamental $\mathrm{TE}_{11}$ mode, and to the excitation of the $\mathrm{TM}_{01}$ mode in the circular waveguide. Moreover, the junction between the rectangular branches and the circular waveguide consists of a Chebyshev-like matching network designed as a cascade of waveguide sections with different heights and lengths. The dimensions of these sections have been optimized to match the WR- 28 waveguide to the MTS input impedance, and are given in Table I. Besides, the length and diameter of the circular waveguide are $\mathrm{H}_{\mathrm{CW}}=1.9 \mathrm{~mm}$ and $\mathrm{D}_{\mathrm{CW}}=8.2 \mathrm{~mm}$, respectively. The $\mathrm{S}_{11}$ obtained with the aforementioned feeding structure is shown in Section V-B.

\section{MANUfACTURING AND EXPERIMENTAL RESUltS}

The RHCP antenna described in Section IV-A has been manufactured and measured.

\section{A. Manufacturing}

In this section, we describe the process followed to manufacture the MTS antenna with a RHCP broadside pencil beam presented in Section IV-A. Fig. 6 shows a picture of

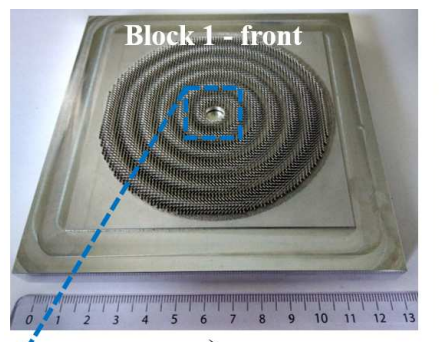

a)

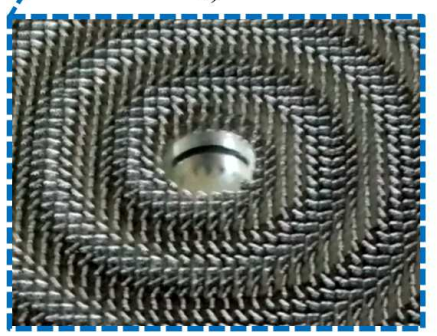

c)

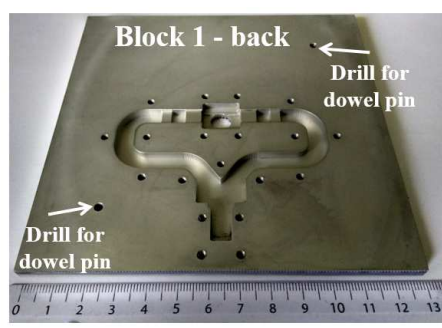

b)

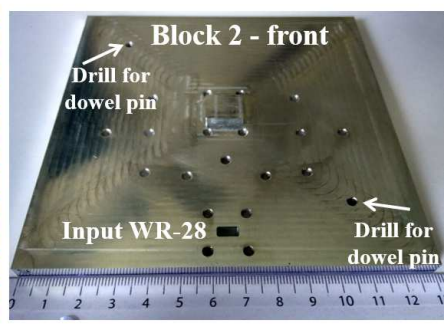

d)
Fig. 6. Picture of the fabricated MTS antenna with RHCP broadside beam. a) Front view of Block-1 with the MTS elements and the circular waveguide feeder. b) Back view of Block-1 with the waveguide divider and the matching sections. c) Zoom to the central region in a). d) Front view of Block-2 with the RW input.

the two prototyped blocks. Block-1, shown in Fig. 6(a), (b) and (c), has been fabricated by a combination of metal additive manufacturing and computer numerical control (CNC) milling in an aluminum base-plate. Indeed, as a first step, the MTS elements have been 3D printed in the front side of the $\mathrm{Al}$ base-plate. To that end, the elliptical cylinders (see Fig. 6(a) and the inset in Fig. 6(c)) have been manufactured using laser beam melting (LBM) with LaserForm AlSi10Mg material on a ProX DMP 320 metal printer [30]. AlSi10Mg alloys typically present an electric conductivity of $2 \times 10^{7} \mathrm{~S} / \mathrm{m}$ [31]-[33]. On the other hand, after evaluating scanning electron microscope (SEM) images of several 3D printed MTS samples, we have determined that the adopted LBM process can guarantee a surface roughness average $R_{a}$ of around $10 \mu \mathrm{m}$.

Once completed the 3D printing of the MTS, we fabricated the waveguides in the back side of the same $\mathrm{Al}$ base-plate by $\mathrm{CNC}$ milling. In Fig. 6(b), one can observe the power divider and the matching sections. One can also notice two drills for the dowel pins used for alignment, and a series of drills to assemble both blocks. On the other hand, Block-2 (see Fig. 6(d)) has been fabricated using conventional CNC milling. It is important to note that, in order to guarantee a good contact between blocks, we have used a contour oversize around the waveguide edges. The adopted approach provides good results in the frequency range of interest, as one can see from the measured reflection coefficient and radiation patterns in the following section.

\section{B. Experimental results}

In the next paragraph, we report the measured reflection coefficient and radiation patterns for the prototype in Fig. 6. First, Fig. 7a presents a comparison between the measured reflection coefficient and the one obtained with CST [25]. An excellent agreement is observed between both curves, 


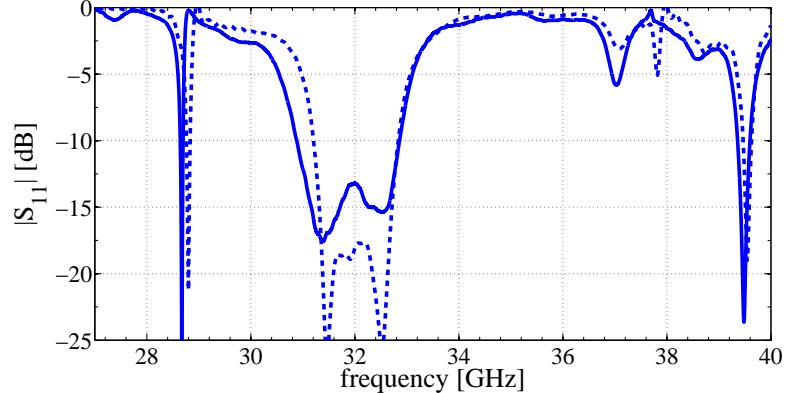

(a)

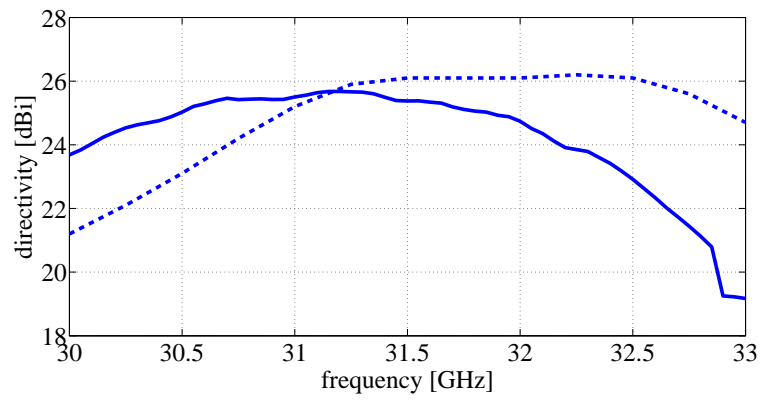

(b)

Fig. 7. (a) Comparison between the reflection coefficient obtained from the CST simulation of the CAD model in Fig. 1 (- - -) and the measurement of the prototype shown in Fig. $6(-)$. (b) Comparison between the simulated $(---)$ and measured (-) broadside directivity in the $30-33 \mathrm{GHz}$ frequency band.

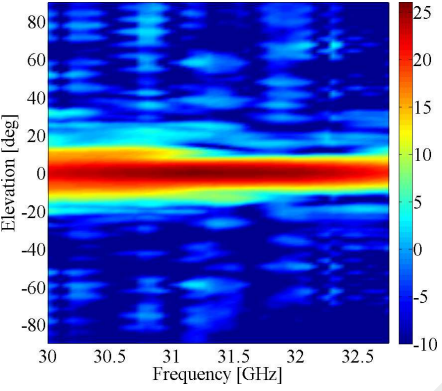

(a)

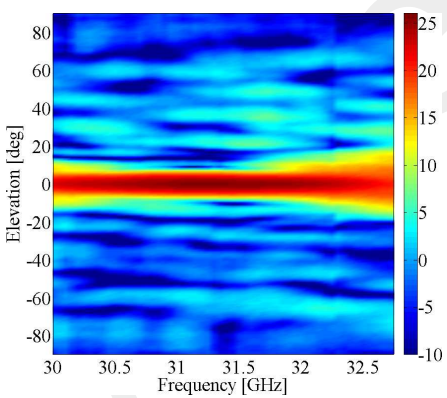

(c)

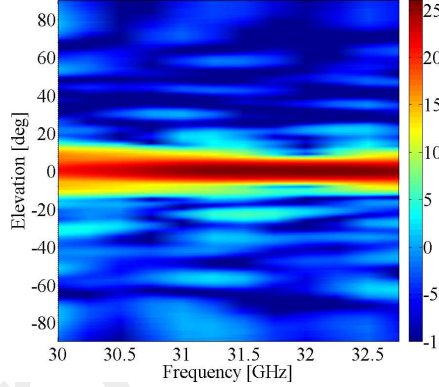

(b)

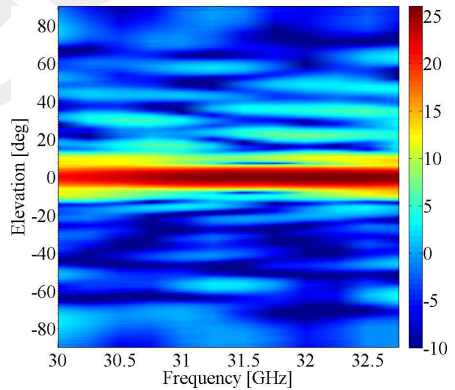

(d)
Fig. 8. (a) Measured and (b) simulated directivity in the $\phi=0^{\circ}$ plane as a function of the frequency and the elevation angle, and (c) measured and (d) simulated directivity in the $\phi=90^{\circ}$ plane, versus frequency and elevation angle.

with the measurements matching even the resonances (around $28.7 \mathrm{GHz}$ and $39.5 \mathrm{GHz}$ ) outside the design frequency range. Despite the slightly higher reflection coefficient measured

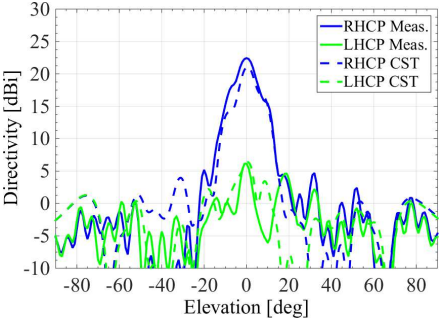

(a)

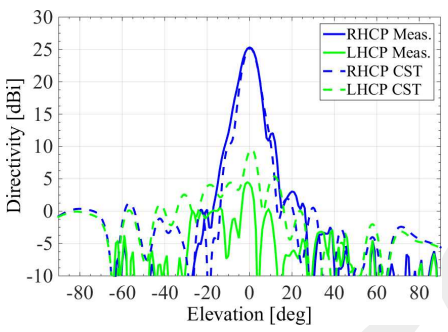

(c)

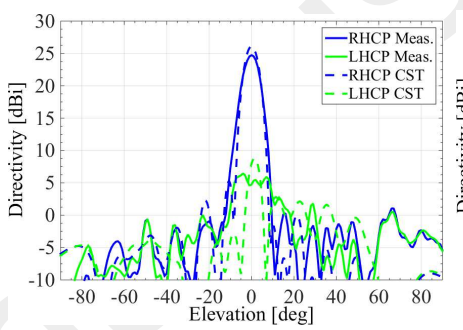

(e)

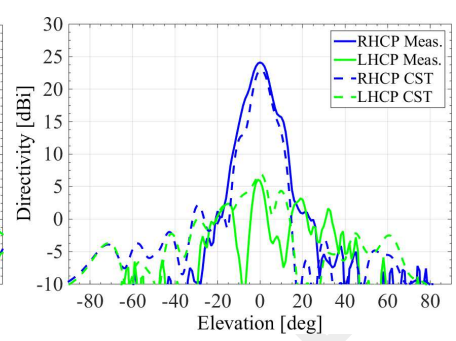

(b)

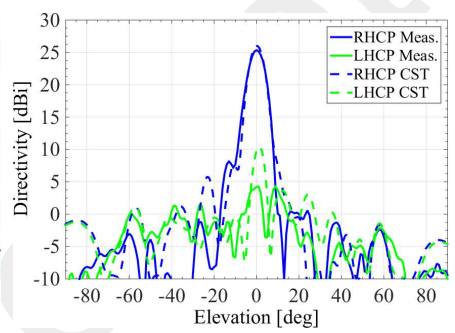

(d)

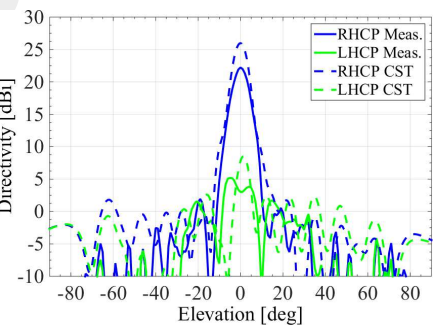

(f)
Fig. 9. Comparison between the simulated (- - -) and measured (-) RHCP directivity patterns, and between the simulated $(---)$ and measured $(-)$ LHCP directivity patterns in the $30-32.5 \mathrm{GHz}$ frequency band. Directivity patterns in the $\phi=0$ plane at (a) $30 \mathrm{GHz}$, (b) $30.5 \mathrm{GHz}$, (c) $31 \mathrm{GHz}$, (d) $31.5 \mathrm{GHz}$, (e) $32 \mathrm{GHz}$, and (f) $32.5 \mathrm{GHz}$.

between $31.2 \mathrm{GHz}$ and $32.8 \mathrm{GHz}$, i.e., in the frequency band of operation, the obtained $\mathrm{S}_{11}$ levels are around $-15 \mathrm{~dB}$ and always well below $-10 \mathrm{~dB}$. One can also observe a small frequency shift towards lower frequencies in the measured $\mathrm{S}_{11}$. This discrepancy is mainly due to the fabrication tolerance in the additive manufacturing process. As detailed next, such tolerance also has an impact on the antenna radiation performance.

Next, we present a comparison between the measured radiation patterns for the structure in Fig. 6 and the ones simulated using CST and the complete CAD model in Fig. 1. Fig. 7b depicts the measured and simulated broadside directivities. Here, one can observe a 3\% frequency shift towards lower frequencies in the peak directivity. Fig. 8(a) and Fig. 8(b) show the measured and simulated directivity in the $\phi=0^{\circ}$ plane, respectively, as a function of the frequency and the elevation angle. In turn, Fig. 8(c) and Fig. 8(d) present the measured and simulated directivity in the $\phi=90^{\circ}$ plane, respectively, versus frequency and elevation angle. One can also observe the shift towards lower frequencies in this figure and a wider measured beam-width in the $\phi=90^{\circ}$ plane above $32.25 \mathrm{GHz}$.

Finally, Fig. 9 shows a comparison between the measured and simulated RHCP and LHCP components of the directivity patterns in the $\phi=0$ plane at $30,30.5,31,31.5,32$ and 
32.5 GHz. In this last figure, one can observe a good agreement between the measured and simulated patterns, except for the slightly different peak levels. This difference is related to the frequency shift observed in Fig. 7b and Fig. 8a. The maximum measured directivity was $25.4 \mathrm{~dB}$ at $31.5 \mathrm{GHz}$, whereas the measured gain at this frequency was $24.4 \mathrm{~dB}$. The $1 \mathrm{~dB}$ loss is due to ohmic losses and inaccuracies in the surface finish.

\section{CONCLUSION}

In this work, we have demonstrated the suitability of a class of metal-only metasurface (MTS) to the design of millimeterwave high-gain antennas. The proposed antenna topology consists of several thousands of sub-wavelength metallic cylinders with different elliptical cross-sections and heights. The cylinders are placed on a ground plane and arranged in a square lattice. This configuration preserves the flatness and lightweight of MTS antennas based on printed patches, and can be easily fabricated by metal additive manufacturing. By using a fully-metallic structure, we can also avoid electrostatic discharge problems, thus simplifying the qualification tests required for space applications. In order to verify the effectiveness of the presented approach, we have designed, manufactured and tested a right-handed circularly polarized antenna with a broadside pencil beam in the Ka band. The measured performance is in good agreement with the theory and the simulation results. The proposed MTS structure may be used in the next generation of antenna systems for space applications. Indeed, the low profile and low mass offered by this antenna makes it an excellent candidate for SmallSats and CubeSats.

\section{ACKNOWLEDGMENT}

The authors are grateful to Xavier Morvan for his help in the fabrication of the prototype and to Dr. Laurent Le Coq for carrying out the antenna measurements.

\section{REFERENCES}

[1] "Nanosatellite \& cubesat database," 2017. [Online]. Available: http://www.nanosats.eu

[2] R. E. Hodges, N. Chahat, D. J. Hoppe, and J. D. Vacchione, "A deployable high-gain antenna bound for Mars: Developing a new foldedpanel reflectarray for the first CubeSat mission to Mars." IEEE Antennas Propag. Mag., vol. 59, no. 2, pp. 39-49, April 2017.

[3] N. Chahat, R. E. Hodges, J. Sauder, M. Thomson, E. Peral, and Y. Rahmat-Samii, "CubeSat deployable Ka-band mesh reflector antenna development for Earth science missions," IEEE Trans. Antennas Propag., vol. 64, no. 6, pp. 2083-2093, Jun. 2016.

[4] C. J. Vourch and T. D. Drysdale, "V-band "bull's eye" antenna for cubesat applications," IEEE Antennas Wireless Propag. Lett., vol. 13, pp. 1092-1095, 2014.

[5] U. Beaskoetxea, S. Maci, M. Navarro-Cía, and M. Beruete, "3-Dprinted $96 \mathrm{GHz}$ bull's-eye antenna with off-axis beaming," IEEE Trans. Antennas Propag., vol. 65, no. 1, pp. 17-25, Jan 2017.

[6] B. Fong, J. Colburn, J. Ottusch, J. Visher, and D. Sievenpiper, "Scalar and tensor holographic artificial impedance surfaces," IEEE Trans. Antennas Propag., vol. 58, no. 10, pp. 3212-3221, Oct. 2010.

[7] A. M. Patel and A. Grbic, "A printed leaky-wave antenna based on a sinusoidally-modulated reactance surface," IEEE Trans. Antennas Propag., vol. 59, no. 6, pp. 2087-2096, Jun. 2011.

[8] G. Minatti, M. Faenzi, E. Martini, F. Caminita, P. De Vita, D. GonzálezOvejero, M. Sabbadini, and S. Maci, "Modulated metasurface antennas for space: Synthesis, analysis and realizations," IEEE Trans. Antennas Propag., vol. 63, no. 4, pp. 1288-1300, Apr. 2015.
[9] S. Pandi, C. A. Balanis, and C. R. Birtcher, "Design of scalar impedance holographic metasurfaces for antenna beam formation with desired polarization," IEEE Trans. Antennas Propag., vol. 63, no. 7, pp. 30163024, Jul. 2015.

[10] G. Minatti, F. Caminita, E. Martini, M. Sabbadini, and S. Maci, "Synthesis of modulated-metasurface antennas with amplitude, phase, and polarization control," IEEE Trans. Antennas Propag., vol. 64, no. 9, pp. 3907-3919, Sept 2016.

[11] D. González-Ovejero, G. Minatti, G. Chattopadhyay, and S. Maci, "Multibeam by metasurface antennas," IEEE Trans. Antennas Propag., vol. 65, no. 6, pp. 2923-2930, Jun. 2017.

[12] G. Minatti, M. Faenzi, M. Mencagli, F. Caminita, D. GonzálezOvejero, C. D. Giovampaola, A. Benini, E. Martini, M. Sabbadini, and S. Maci, "Metasurface antennas," in Aperture Antennas for Millimeter and Sub-Millimeter Wave Applications, A. Boriskin and R. Sauleau, Eds. Springer, 2018, ch. 9, pp. 289-333.

[13] N. Chamberlain, J. Chen, P. Focardi, R. Hodges, R. Hughes, J. Jakoboski, J. Venkatesan, and M. Zawadzki, "Juno microwave radiometer patch array antennas," in Proc. IEEE Antennas Propag. Soc. Int. Symp., Charleston, SC, Jun. 1-5 2009, pp. 1-4.

[14] N. Chahat, B. Cook, H. Lim, and P. Estabrook, "All-metal dual frequency rhcp high gain antenna for a potential europa lander," IEEE Trans. Antennas Propag., submitted to.

[15] D. González-Ovejero, T. J. Reck, C. D. Jung-Kubiak, M. AlonsoDelPino, and G. Chattopadhyay, "A class of silicon micromachined metasurface for the design of high-gain terahertz antennas," in Proc. IEEE Antennas Propag. Soc. Int. Symp., Fajardo, Puerto Rico, Jun. 26 - Jul. 1 2016, pp. 1191-1192.

[16] D. González-Ovejero, C. Jung-Kubiak, M. Alonso-delPino, T. Reck, and G. Chattopadhyay, "Design, fabrication and testing of a modulated metasurface antenna at $300 \mathrm{GHz}$," in Proc. 11th Eur. Conf. Antennas Propag. (EUCAP), Paris, France, Mar. 19-24 2017, pp. 3416-3418.

[17] F. Puggelli, G. Minatti, Y. Vardaxoglou, and S. Maci, "Design of a modulated dielectric metasurface antenna," in Loughborough Antennas Propag. Conf. (LAPC), Loughborough, UK, Nov. 2-3 2015, pp. 1-3.

[18] S. Zhang, R. K. Arya, S. Pandey, Y. Vardaxoglou, W. Whittow, and R. Mittra, "3D-printed planar graded index lenses," IET Microw., Antennas Propag., vol. 10, no. 13, pp. 1411-1419, 2016.

[19] A. W. Glisson, "Electromagnetic scattering by arbitrarily shaped surfaces with impedance boundary condition," Radio Sci., vol. 27, no. 6, pp. 935943, Nov. 1992.

[20] E. Bleszynski, M. Bleszynski, and T. Jaroszewicz, "Surface-integral equations for electromagnetic scattering from impenetrable and penetrable sheets," IEEE Antennas Propag. Mag., vol. 35, no. 6, pp. 14-25, Dec. 1993.

[21] S. Tretyakov, Analytical modeling in applied electromagnetics. Norwood, MA: Artech House, 2003.

[22] C. Holloway, E. F. Kuester, J. Gordon, J. O'Hara, J. Booth, and D. Smith, "An overview of the theory and applications of metasurfaces: The two-dimensional equivalents of metamaterials," IEEE Antennas Propag. Mag., vol. 54, no. 2, pp. 10-35, Apr. 2012.

[23] E. F. Kuester, M. Mohamed, M. Piket-May, and C. Holloway, "Averaged transition conditions for electromagnetic fields at a metafilm," IEEE Trans. Antennas Propag., vol. 51, no. 10, pp. 2641-2651, Oct. 2003.

[24] G. Minatti, E. Martini, and S. Maci, "Efficiency of metasurface antennas," IEEE Trans. Antennas Propag., vol. 65, no. 4, pp. 1532-1541, April 2017

[25] CST of America, "CST Microwave Studio," Anaheim, CA, 2016.

[26] M. Mencagli, C. D. Giovampaola, and S. Maci, "A closed-form representation of isofrequency dispersion curve and group velocity for surface waves supported by anisotropic and spatially dispersive metasurfaces," IEEE Trans. Antennas Propag., vol. 64, no. 6, pp. 2319-2327, Jun. 2016.

[27] H. Bilow, "Guided waves on a planar tensor impedance surface," IEEE Trans. Antennas Propag., vol. 51, no. 10, pp. 2788-2792, Oct. 2003.

[28] A. M. Patel and A. Grbic, "Effective surface impedance of a printedcircuit tensor impedance surface (PCTIS)," IEEE Trans. Microw. Theory Tech., vol. 61, no. 4, pp. 1403-1413, April 2013.

[29] "Frequency and channel assignments, Module 201, Rev. C," Jet Propulsion Laboratory, California Institute of Technology, Dec. 2014. [Online]. Available: https://deepspace.jpl.nasa.gov/files/810-005/ 201/201C.pdf

[30] "3D Systems Customer Innovation Center (CIC)," Leuven, Belgium, 2017. [Online]. Available: https://www.3dsystems.com

[31] R. Brandt and G. Neuer, "Electrical resistivity and thermal conductivity of pure aluminum and aluminum alloys up to and above the melting temperature," Int. J. Thermophys., vol. 28, no. 5, pp. 1429-1446, Oct. 2007. 
[32] P. Uliasz, T. Knych, M. Piwowarska, and J. Wiechec, "The influence of heat treatment parameters on the electrical conductivity of AlSi7Mg and AlSi10Mg aluminum cast alloys," in 13th Int. Conf. Aluminum Alloys, Pittsburgh, PA, June 2012, pp. 129-135.

[33] C. Silbernagel, I. Ashcroft, P. Dickens, and M. Galea, "Electrical resistivity of additively manufactured $\mathrm{AlSi} 10 \mathrm{Mg}$ for use in electric motors," Additive Manufacturing, vol. 21, pp. 395-403, Mar. 2018. 


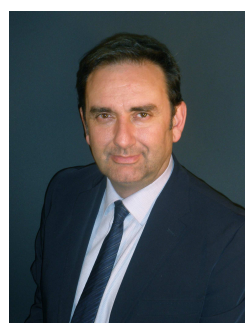

Stefano Maci (M'92-SM'99-F'04) received the Laurea degree (cum laude) in electronics engineering from the University of Florence, Florence, Italy, in 1987.

Since 1997, he has been a Professor with the University of Siena, Siena, Italy. His research interests include high-frequency and beam representation methods, computational electromagnetics, large phased arrays, planar antennas, reflector antennas and feeds, metamaterials, and metasurfaces. His research activity is documented in 150 papers published in international journals, among which 100 are in IEEE journals, 10 book chapters, and about 400 papers in international proceedings. These papers have received around 5700 citations.

Prof. Maci was a recipient of the European Association on Antennas and Propagation (EurAAP) Award in 2014, and the Sergei A. Schelkunoff Transactions Prize Paper Award and the Chen-To Tai Distinguished Educator Award, both from the IEEE Antennas and Propagation Society (IEEE AP-S) in 2016. Since 2000, he has been a member of the Technical Advisory Board of 11 international conferences and the Review Board of 6 International Journals. He has organized 25 special sessions in international conferences and held 10 short courses in the IEEE AP-S Symposia about metamaterials, antennas, and computational electromagnetics. In 2004, he was the founder of the European School of Antennas (ESoA), a post graduate school that presently comprises 30 courses on antennas, propagation, electromagnetic theory, and computational electromagnetics, with 150 teachers from 15 countries. From 2004 to 2007, he was WP Leader of the Antenna Center of Excellence (ACE, FP6-EU), and from 2007 to 2010 he was the International Coordinator of a 24institution consortium of a Marie Curie Action (FP6). Since 2010, he has been the Principal Investigator of 6 cooperative projects financed by the European Space Agency. He has been Director of the University of Siena's Ph.D. program in information engineering and mathematics from 2008 to 2015, and a member of the National Italian Committee for Qualification to Professor from 2013 to 2015. He is the Director of the consortium FORESEEN, presently involving 48 European institutions, and principal investigator of the Future Emerging Technology project "Nanoarchitectronics" of the 8th EU Framework program. He was co-founder of 2 spin-off companies. He is a Distinguished Lecturer of the IEEE AP-S. He was a former member of the IEEE AP-S AdCom, EurAAP Board of Directors, and Antennas and Propagation Executive Board of the Institution of Engineering and Technology (IET, UK). He was an Associate Editor of IEEE Transactions on Antennas and Propagation and Chair of the Award Committee of IEEE AP-S.

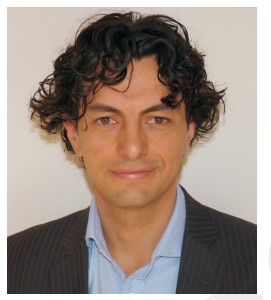

Mauro Ettorre (M'08-SM'15) received the Laurea degree "summa cum laude" in Electrical Engineering and a Ph.D. in Electromagnetics from the University of Siena, Italy, in 2004 and 2008, respectively. Part of his Ph.D. work was developed at the Netherlands Organisation for Applied Scientific Research (TNO), The Netherlands, where he later worked as an Antenna Researcher.

From 2008 to 2010, Dr. Ettorre was a Postdoctoral Fellow at the Institut d'Electronique et de Télécommunications de Rennes (IETR), Université de Rennes 1, France. In 2010 and 2016, he was a Visiting Scholar with the Radiation Laboratory, Department of Electrical Engineering and Computer Science, University of Michigan, Ann Arbor, MI, USA. Since October 2010 he is a Research Scientist at the French National Center for Scientific Research (CNRS), within the IETR. In 2014, he assumed responsibilities for the multibeam antenna activity for satellite applications in the joint laboratory between IETR and Thales Alenia Space, Toulouse, France. In 2015, he was an invited professor at Tokyo Institute of Technology, Japan.

Dr. Ettorre's research interests include the analysis and design of leakywave antennas, periodic structures, millimeter-wave antennas, non-diffractive radiation, near-field focusing techniques, and wireless power transfer systems.

Dr. Ettorre received the Young Antenna Engineer Prize at the 2008 European Space Agency Antenna Workshop in The Netherlands and the Best Paper Award in Electromagnetics and Antenna Theory at the 2018 European Conference on Antennas and Propagation (EuCAP), London, UK. 